ESCHARECTOMY has been shown to improve the survival rates and the outcomes in burns. This observational study was conducted to assess the role of escharectomy on the inflammatory mediators in major burns. Seventeen ASA physical status II or status III adult surviving major burn patients were recruited. When the escharectomy was scheduled, a series of blood samples was obtained at -3 and -1 days preoperation, and +1 and +3 postoperation. The changing levels of endotoxin, cytokines, and adhesion molecules were measured with a quantitative sandwich immunoassay. Extensive escharectomy did not appear to have any significant impact on the levels of tumor necrosis factor alpha, interleukin-10, soluble intracellular adhesion molecule-1 and soluble vascular adhesion molecule-1. Meanwhile, endotoxin and E-selectin were significantly decreased after escharectomy. Escharectomy appeared to have a limited immunomodulatory effect on the inflammatory mediators in systemic inflammatory responses induced by major burns. This is probably related to the timing and extent of surgery, and the complex nature of burn-related inflammation.

Key words: E-selectin, Interleukin-10, soluble intercellular adhesion molecule-1, Thermal injury, Tumor necrosis factor alpha, Soluble vascular adhesion molecule-1, Burn wound excision

\section{The limited immunomodulatory effects of escharectomy on the kinetics of endotoxin, cytokines, and adhesion molecules in major burns}

\author{
Tae-Hyung Han ${ }^{\text {CA }}$, Soo-Yeon Lee, Jung-Eun Kwon, \\ In-Suk Kwak and Kwang-Min Kim
}

Department of Anesthesiology and Pain Medicine, Hangang Sacred Heart Hospital Hallym University, College of Medicine, 94-200 Yongdungpo-Dong, Yongdungpo-Ku, Seoul 150-710, Korea

\author{
${ }^{\mathrm{CA}}$ Corresponding Author \\ Tel: +82226395500 \\ Fax: + 82226314387 \\ E-mail: athan@unitel.co.kr
}

\section{Introduction}

The patho-physiological events following the immense tissue damage of major burn injury include the surface effects of the heat on superficial tissue, but also should be viewed as an acute systemic illness, ${ }^{1,2}$ initiated by a number of processes such as the development of ischemia-reperfusion injury related to burn shock and resuscitation as well as the presence of endotoxemia. ${ }^{3}$ Major burns produce both local and systemic disturbances due to the release of inflammatory mediators, depending upon the type and extent of burn and the time elapsed since the injury. ${ }^{4}$

The acute inflammatory response results from an integration of a complex cascade of events, including vasodilation, increased vascular permeability, and exudation of fluid and plasma protein. The rapid overproduction of inflammatory active substances, leukocyte activation, activation of endothelial cells, and expression of adhesion molecules and induction of nitric oxide synthase all lead to systemic inflammatory response syndrome (SIRS), resulting in progressive multi-organ failure and death..$^{5-7}$

Many experimental and clinical studies have demonstrated a pronounced immunosuppression following major burn injury. ${ }^{8}$ Since burn eschar is thought to be the source of denatured proteins, mediators, and toxins, which cause immunosuppression and infection, prompt excision of burn eschar could be an effective way to avoid absorption of immunosuppressive substances. ${ }^{9}$ Removing the dead devitalized tissue and grafting would not only remove the source of infection, but also decrease the release of inflammatory mediators, and improve survival, prevent postburn immunosuppression and maintain skin barrier function. ${ }^{10}$ Experimentally, although some specific parameters of immunity may be improved by early excision and wound closure, ${ }^{11}$ little defined physiologic benefit has been proved.

A variety of inflammatory mediators such as endotoxin, complement fragments, cytokines, adhesion molecules and lipid mediators are generated shortly after the burn injury at the site of the initial lesion and serve to recruit inflammatory cells at the later stages. In the present study, we measured the dynamic changes of the levels of endotoxin, proinflammatory and anti-inflammatory cytokines, and soluble adhesion molecules, and compared their relations before and after extensive escharectomy. Attempts were made to better understand the immunological role of extensive early escharectomy in preventing SIRS. 


\section{Materials and methods}

The protocol was reviewed and approved by the institutional ethics committee for human study, and written informed consent was obtained before the study from each patient or legal guardian if mentally incompetent.

When a major burn patient with possible candidacy for the study arrived in the emergency department, the anesthesia department was notified for the potential study subject identification. As the patients were scheduled for the escharectomy, the primary investigator was informed at least 3 days before.

At this time, patients were selected based upon their clinical status, and those with inhalational injury, SIRS, sepsis, septic shock, adult respiratory distress syndrome and multiple organ dysfunction syndromes were included. The guidelines were based on the criteria of the College of Chest Physicians/Society of Critical Care Medicine. ${ }^{12,13}$

Patients who suffered from any type of immune deficiency syndrome, had taken any immune therapy, steroids or anticancer drug, any hormonal medications or who had immune system disease were excluded from the study. Since it has been well known that non-survivors have an exponential increase in the inflammatory mediators, concerns were raised that those results might falsely elevate the levels. Therefore, samples from those patients were excluded in the final analysis.

On recruitment, an arterial line for multiple sampling was placed in either the upper or lower extremity, in the intensive care unit, and locked with heparin. Serial collections were performed four times between 8:00 and 9:00 a.m. at 72 and $24 \mathrm{~h}$ before and 24 and $72 \mathrm{~h}$ after the surgery. Three milliliters of blood specimens were collected in endotoxin-free ethylenediamine tetraacetic acid-containing blood specimen tubes (BD Vacutainer ${ }^{\mathrm{TM}}$; BD Vacutainer Systems, Plymouth, UK). These were immediately centrifuged at $3000 \mathrm{rpm}$ for $60 \mathrm{sec}$ within $30 \mathrm{~min}$ of blood sampling, and platelet-rich plasma was obtained and stored in aliquots at $-70^{\circ} \mathrm{C}$ until the later combined assay. These samples were kept in the refrigerator until each individual patient's eventual outcome was determined. All the samples were analyzed at one time, approximately 3 months after the collections were completed.

Anesthesia was induced by intravenous sodium thiopental, vecuronium and fentanyl, and was maintained by continuous infusion of fentanyl, nitrous oxide and oxygen, and sevoflurane. Intermittent boluses of vecuronium provided muscle relaxation.

Endotoxin levels were measured by the chromogenic limulus amebocyte lysate (LAL) test (QCL $1000^{\circledR}$; Biowhittaker, East Rutherford, NJ, USA), which is a quantitative test for Gram-negative bacter- ial endotoxin. A sample is mixed with the LAL supplied in the test kit and incubated at $37^{\circ} \mathrm{C}$ for 10 min. A substrate solution is then mixed with the LAL sample and incubated at $37^{\circ} \mathrm{C}$ for an additional $6 \mathrm{~min}$. The reaction is stopped with stop reagent. If endotoxin is present in the sample, a yellow color will develop. The absorbance of the sample can be determined spectrophotometrically at 405-410 nm. Since this absorbance is in direct proportion to the amount of endotoxin present, the concentration of endotoxin can be calculated from a standard curve.

Cytokines of tumor necrosis factor alpha (TNF- $\alpha$ ) and interleukin-10 (IL-10) were measured with quantitative sandwich enzyme-linked immunosorbent assay (ELISA) test kits (Quantikine ${ }^{\circledR} ;$ R \& D systems, Inc., Minneapolis, MN, USA) according to instructions given by the manufacturer and optical densitometry. These assays did not measure biological activity, but rather quantitative assessment of immunoreactivity is provided. The minimum detectable doses of TNF- $\alpha$ and IL-10 with these kits were 4 and $3.9 \mathrm{pg} / \mathrm{ml}$.

Three adhesion molecules of soluble intracellular adhesion molecule-1 (sICAM-1), soluble vascular adhesion molecule-1 (sVCAM-1), and E-selectin were all measured similarly with the same quantitative sandwich ELISA test kit and optical densitometry. The minimum detectable doses of sICAM-1, sVCAM-1 and E-selectin were $0.35,0.6$, and $0.5 \mathrm{pg} / \mathrm{ml}$, respectively.

Measured parameters included the demographic and surgical data such as age, gender, height and weight, burn type and percent of total body surface area, as well as American Society of Anesthesiologists physical status classification.

The SPSS program (version 10.01; SPSS Inc., Chicago, IL, USA) was used for statistical analysis and all values are expressed as mean \pm standard deviation. Demographic and surgical data were summarized by descriptive analysis. The level of changes of endotoxin, cytokines, adhesion molecules and E-selectin were individually determined with repeated-measures of analysis of variance (ANOVA) with Bonferroni's correction. Each patient served as their own control, values were compared before and after the escharectomy. $p<0.05$ was considered statistically significant. Pearson's correlations were performed to delineate the relationship between the various inflammatory mediators.

\section{Results}

A total of 25 patients were recruited, and from these three expired within 1 week after the escharectomy and another five suffered from major complications described as follows before or after surgery. Those samples were exempted from final analysis. The 
remainder was 17 major burn patients with a total body surface area of $25 \%$ or more, who survived more than 3 months after blood sampling, and from whom blood specimens could be repeatedly obtained. Every other day samples from those patients were finally analyzed with ELISA.

The patients' characteristics and surgical data are summarized in Table 1. Most suffered from extensive major burns. The removal of dead devitalized tissue was performed approximately within 1 week.

Figure 1 shows the changes of endotoxin levels perioperatively. Statistically significant differences were noted before and after escharectomy.

Figure 2 shows the changes of TNF- $\alpha$ and IL-10. In both groups, only day +1 showed a significant decrease in their levels. These were increased at +3 days, following the increasing trend again. Interestingly, the level would have been thought to be there anyway even without the scheduled escharectomy.

Figure 3 shows the changes of adhesion molecules. sICAM-1 showed a continued increasing trend regardless of escharectomy compared with the baseline. In contrast, sVCAM-1 decreased even before the surgery, and remained as such during the rest of study period regardless of the extensive escharectomy. It was even slightly increased after the procedure. E-selectin had a statistically meaningful decrease after the extensive escharectomy and remained at a lower level compared with the baseline at +3 days.

Pearson's correlation analysis revealed statistically significant high correlations between endotoxin and E-selectin, as well as between TNF- $\alpha$ and IL-10.

\section{Discussion}

In this study, we have shown that the immune modulatory effect of escharectomy on the kinetics of endotoxin, cytokines, and adhesion molecules in major burns appeared to be limited, in that it did not change that much over a matter of days. In essence, our data did not demonstrate that the removal of the

Table 1. Patient characteristics, American Society of Anesthesiologists (ASA) physical status classification, and burn data

\begin{tabular}{lc}
\hline Characteristic & Value \\
\hline Age (years) & $40.9 \pm 9.3$ \\
Sex (male/female) & $12 / 5$ \\
Weight (kg) & $60.5 \pm 4.8$ \\
Height (cm) & $160.7 \pm 5.6$ \\
ASA class (II/III) & $3 / 14$ \\
Total body surface area (\%) & $42.9 \pm 17.0$ \\
Elapsed days since initial injury until & $5.3 \pm 1.7$ \\
$\quad$ escharectomy & \\
\hline
\end{tabular}

Values presented as mean \pm standard deviation.

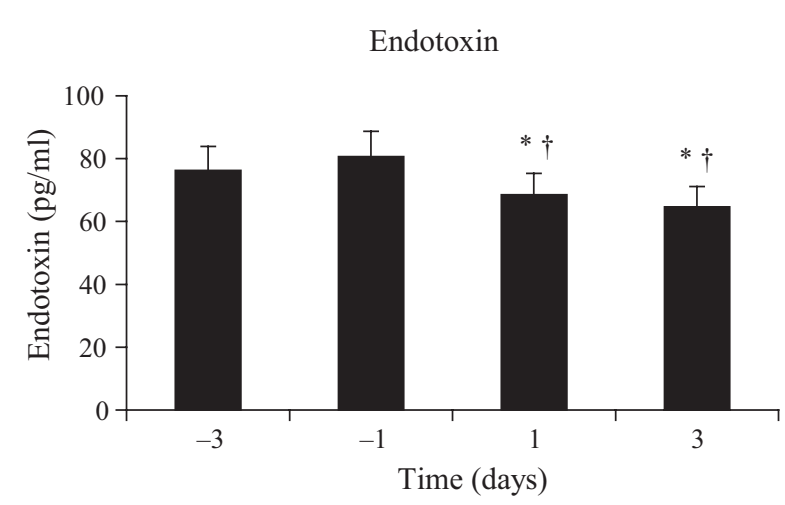

FIG. 1. Bar graph illustrating the changing levels of endotoxin before and after the extensive escharectomy in major burns (repeated-measures ANOVA, ${ }^{*} p<0.05$ when -3 days compared with $-1,+1$ and +3 days, ${ }^{\dagger} p<0.05$ when -1 day compared with +1 and +3 days).

burn has much of a profound effect. Although we found that values of endotoxin and E-selectin decreased significantly after escharectomy, it probably has no clinically significance. In spite of these findings, it cannot be precluded that early removal of the burn does not affect the patient's ability to survive or resist infection.

A number of reasons should be considered to explain the limited, minimal responses to escharectomy in our study. The patient's selection issue should be considered at first. Only the samples taken from survivors were used for the final analysis. Perhaps the
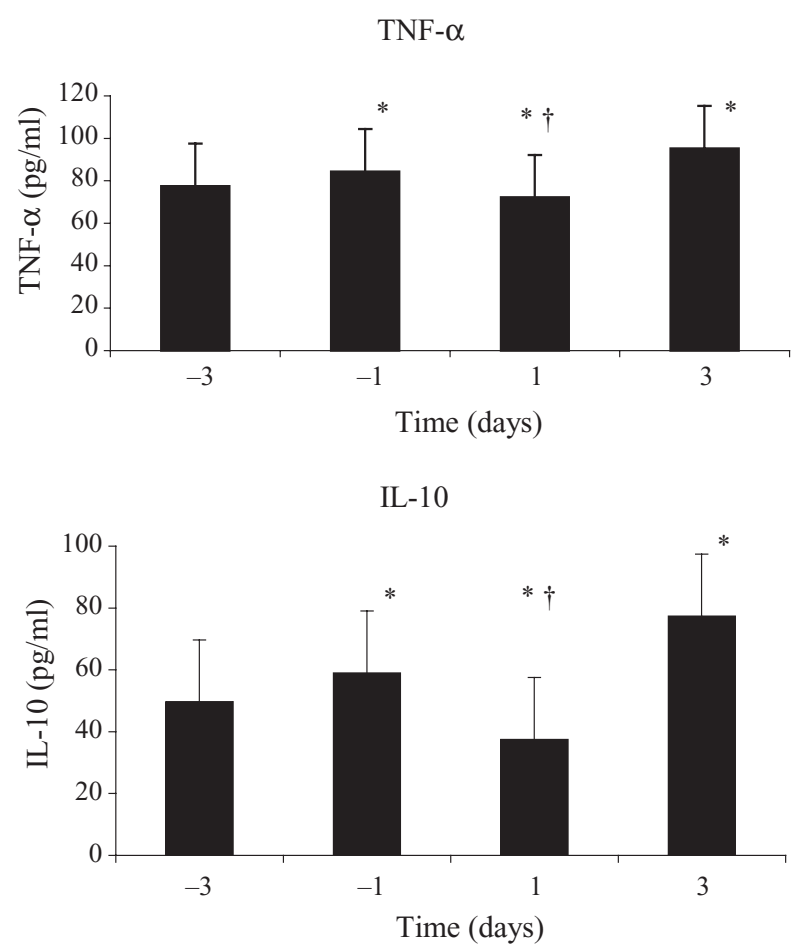

FIG. 2. Bar graphs illustrating the changes of TNF- $\alpha$ and IL-10 before and after extensive escharectomy in major burns (repeated-measures ANOVA, ${ }^{*} p<0.05$ when -3 days compared with $-1,+1$ and +3 days, ${ }^{\dagger} p<0.05$ when +1 day compared with -1 and +3 days). 

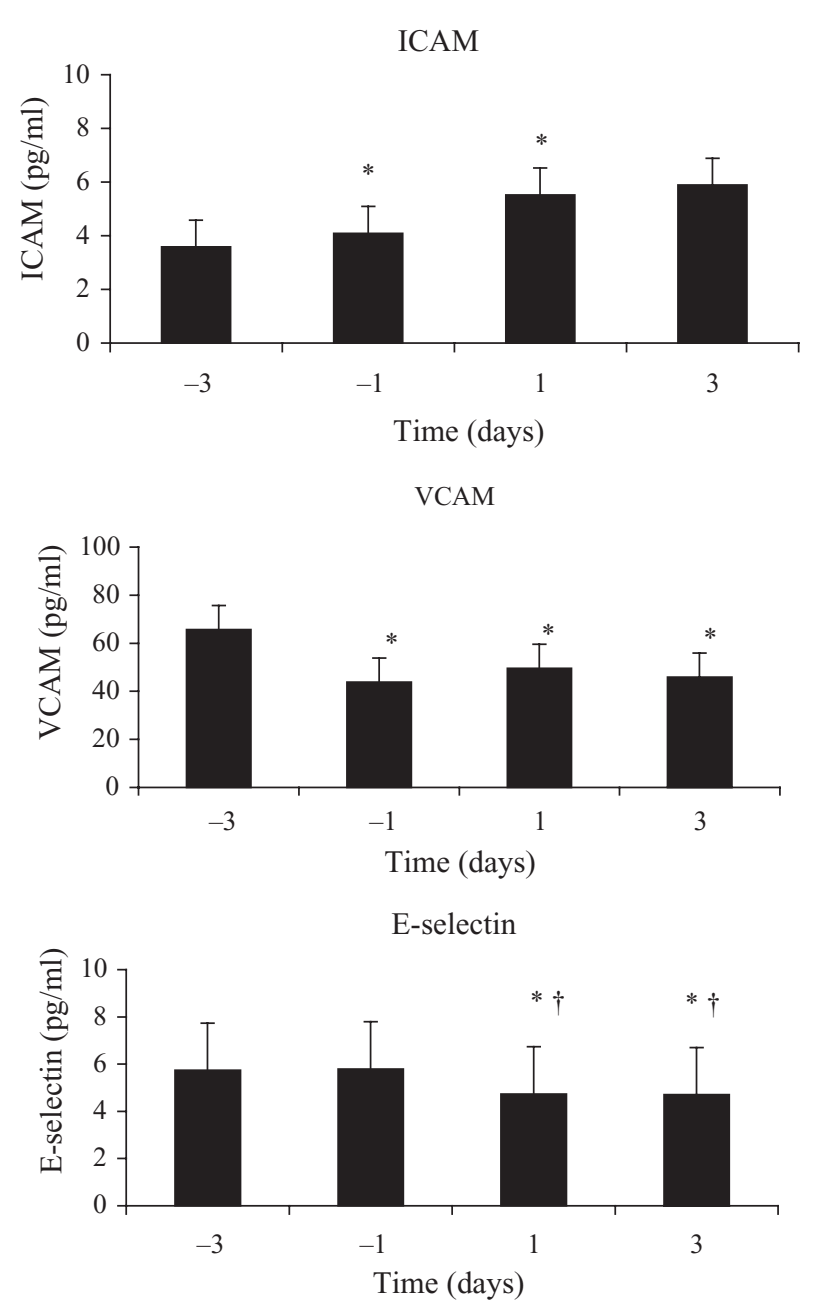

FIG. 3. Bar graphs illustrating the changes of sICAM-1, sVCAM-1, and E-selectin before and after extensive escharectomy in major burns (repeated-measures ANOVA, ${ }^{*} p<$ 0.05 when -3 days compared with $-1,+1$ and +3 days, $p<0.05$ when +1 day compared with -1 and +3 days).

patients were not stressed enough. Possibly, the burns were too small. The immune responses for the non-survivors have been shown to be dramatic. ${ }^{14}$ We intentionally excluded these patients since their results could have falsely elevated the results and may have masked the true effect of escharectomy. It was not surprising that the changing trend was somewhat less than impressive.

Second, the timing and extent of surgery might have been another factor. We considered a few days before and after the burn excision. This may not be enough time to see a difference. It frequently takes more time for inflammatory mediators and the stress response to resolve. In our burn center, it is customary to perform escharectomy at approximately 5 days (3-7 days) after initial thermal injury and the burn shock stage. Furthermore, removing dead and devitalized tissue is limited and partial, and carried out in multiple staged operations. It had been previously suggested that prompt escharectomy could reduce the production and release of inflam- matory cytokines, and lessen the myocardial injury induced by uncontrolled inflammatory response. Escharectomy en masse during the shock stage could weaken the ability of burn sera to induce the secretion of inflammatory cytokines by macrophages. It was therefore beneficial for the prevention of the development of postburn systemic inflammatory response syndrome. ${ }^{11}$ The likelihood is high that when the removal of dead devitalized tissue is delayed to after the burn shock stage, the systemic release of inflammatory mediators from the eschar has already been initiated and settled in the systemic circulation. Accordingly, escharectomy performed 5 days after the burn trauma as in our study would not significantly affect the levels of inflammatory mediators.

Clinically and immunologically, many factors other than escharectomy should help burn patients. The use of antibiotics, daily wound dressing, immediate fluid resuscitation, adequate nutritional support, management of pulmonary complications and good covering of the burn wounds have all contributed to the recuperation of burn patients and improve immunologically. Postoperatively, the patients still have donor sites that need to heal, so in essence the wound is not closed.

Third, the general properties of cytokines and other inflammatory mediators are very vulnerable and relatively non-specific, such that production is dependent on the status of the factories, especially macrophages. The potential for a self-perpetuating process, because of a lack of a negative feedback loop, can explain the progression of inflammation, sepsis and organ failure, even in the absence of continuing injury or infection. The level of circulating cytokines often does not necessarily correlate with the degree of clinical illness and tissue activity. This is because the signal to release the secondary mediators is initiated by the cytokines, and the continued presence of the signal is no longer required. In addition, the action of the cytokines is either at the cell surface receptor or inside the cell, and circulating levels often do not reflect the intracellular levels. The patients also have much more pain postoperatively, which may also turn on cytokine expression. Measuring the cytokines after total healing may make a difference. For these reasons, it is not surprising that circulating levels of many of the cytokines often do not necessarily reflect the clinical status of critically ill patients, including those with sepsis and burns. ${ }^{15}$

Fourth, a variety of inflammatory mediators in trauma, sepsis and burns interact in a complex manner to produce the disease state. Multiple alterations in inflammatory and immunologic function have been demonstrated in clinical and experimental situations after trauma and hemorrhage; in particular, the activation of various humoral (e.g. complement, coagulation) and cellular systems (neutrophils, 
endothelial cells, macrophages). ${ }^{16}$ As a consequence of this activation process, there is synthesis, expression and release of numerous mediators (toxic oxygen species, proteolytic enzymes, adhesion molecules, cytokines), which may produce a generalized inflammation and tissue damage in the body. Mediators are responsible for ongoing interactions of different cell types and for amplification effects through their networks and feedback cycles, finally leading to sustained inflammation and multiple organ damage in the body. In the setting of trauma and shock, many activators including bacterial as well as non-bacterial factors may be present that induce local and systemic inflammatory responses. Although the potential role of bacteria and endotoxin translocation and its clinical relevance remains controversial, many lines of evidence support the concept that the gut may be the reservoir for systemic sepsis and subsequent multiple-organ failure in a number of pathophysiologic states. ${ }^{17}$ Many of the problems that burn doctors deal with come with a secondary infection, not with the initial insult. If we measured the levels after sepsis then we might find a more pronounced difference. Most people are not willing to wait until patients become sick before they remove the burn. We all remove it within 1 week, so our patients do pretty well.

\section{Endotoxin}

In burns, endotoxin values were high in the poor outcome group, serving as the trigger for evolution of burn-related pathology. ${ }^{18}$ Escharectomy during burn shock was shown to effectively eliminate the effects of the endotoxemia, thereby preventing the accentuated response to endotoxin that is seen when the burn wound is left intact, even if it is uninfected. ${ }^{19}$

\section{Cytokines}

There is a growing interest in determining the role of TNF- $\alpha$ in burns since an intense immuno-inflammatory reaction is expected to take place in septic shock, severe sepsis and trauma, including major burns patients. Many suggest that the beneficial effects of early extensive burn wound excision and grafting are likely to be related to the normalization of increased macrophage $\mathrm{TNF}-\alpha$ production, the amelioration of hypermetabolism with sepsis, as well as the maintenance of skin barrier function. 9,20,21

We were able to show a temporary TNF- $\alpha$ decrease to escharectomy, which returned to its increasing trend again within $72 \mathrm{~h}$ at a much higher level than the baseline. This is probably due to the relatively non-specific nature of cytokine response to stress. Unless the devitalized dead tissue is to be removed in en masse at the early stage of injury, its effect in TNF- $\alpha$ would probably be minimal. ${ }^{11}$ In addition, clinical deterioration after burn wound dressing or debridement may be related to predominantly the systemic release of IL- 6 , instead of TNF- $\alpha .^{22}$

IL-10 exerts a number of anti-inflammatory effects, including regulation and inhibition of the synthesis of the inflammatory cytokines and immune responses. The production of IL-10 seems to be predominantly stimulated by TNF- $\alpha$. Hence, they will be likely to have similar kinetic profiles with TNF- $\alpha$. Increased levels of IL-10 have been detected during cardiac surgery after an increase in the proinflammatory cytokines, such as TNF- $\alpha$, and may represent an endogenous response to limit the inflammatory response. $^{23}$

\section{Adhesion molecules}

The importance of early escharectomy during the burn shock period has been previously emphasized by the prevention of expression of adhesion molecules including sICAM-1 and E-selectin mRNA and SIRS. ${ }^{11}$ Our results showed that sICAM-1 increased regardless of the performance of escharectomy. This can be attributed to the time and extent of surgery, in that ours was a partial, delayed procedure although it was extensive.

After a wound revision in severe burns, the patients may show signs of sepsis, a transient endotoxemia, and increased expression of the $\beta_{2}$ integrin CD11b, as well as elevated plasma levels of soluble E-selectin, indicating its close association to systemic inflammation. ${ }^{24}$ On the contrary to sICAM-1, however, E-selectin is a late responder of the inflammation, which might explain the better response to escharectomy performed later stage in our patients. $^{25}$

In conclusion, massive tissue trauma causes immediate activation of endotoxin, selected chemokines and adhesion molecules within minutes of the injury, which will then persist depending on the type and severity of the injury for a substantial length of time. The timing and extent of removal of dead devitalized tissue is the most influencing factor in modulating immune mediators. Therefore, the importance of early en masse burn wound excision during the burn shock stage should be clinically emphasized in major burn patients to maximize its benefits, such as decreased susceptibility to complications or improvement of outcome.

ACKNOWLEDGEMENT. Supported by a grant number 01-2003-01 from the Hallym University Medical Center Research Fund.

\section{References}

1. Munster AM. The immunological response and strategies for intervention. Total Burn Care, 2nd edn, Philadelphia, PA: W.B. Saunders Co., 1996: $297-392$. 
2. Sparkes BG. Immunological responses to thermal injury. Burns 1997; 23: $106-113$.

3. Tadros T, Traber DL, Heggers JP, Herndon DN. Effects of interleukin1alpha administration on intestinal ischemia and reperfusion injury, mucosal permeability, and bacterial translocation in burn and sepsis. Ann Surg 2003; 237: 101-109.

4. Yamada Y, Endo S, Inada K, et al. Tumor necrosis factor- $\alpha$ and tumor necrosis factor receptor I, II levels in patients with severe burns. Burns 2000; 26: 239-244.

5. Cumming J, Purdue GF, Hunt JL, O'Keefe GE. Objective estimates of the incidence and consequences of multiple organ dysfunction and sepsis after burn trauma. J Trauma 2001; 50: 510-515.

6. Ravindranath T, Al-Ghoul W, Namak S, et al. Effects of burn with and without Escherichia coli infection in rats on intestinal vs. splenic T-cell responses. Crit Care Med 2001; 29: 2245-2250.

7. Hall RI, Smith MS, Rocker G. The systemic inflammatory responses to cardiopulmonary bypass: pathophysiological, therapeutic and pharmacological considerations. Anesth Analg 1997; 85: 766-782.

8. Hansbrough JF, Zapata-Sirvent R, Hoyt D. Postburn immune suppression: an inflammatory response to the burn wound? J Trauma 1990; 30: $671-674$.

9. Schwacha MG, Knoferl NW, Chaudry IH. Does burn wound excision after thermal injury attenuate subsequent macrophage hyperactivity and immunosuppression? Shock 2000; 14: 623-628.

10. Yamamoto H, Siltharm S, deSerres S, Hultman CS, Meyer AA. Immediate burn wound excision restores antibody synthesis to bacterial antigen. $J$ Surg Res 1996; 63: 157-162.

11. Huang Y, Yang Z, Chen F, Crowther RS, Li A. Effects of early eschar excision en masse at one operation for prevention and treatment of organ dysfunction in severely burned patients. World J Surg 1999; 23: $1272-1278$.

12. Members of the American College of Chest Physicians/Society of Critical Care Medicine Consensus Conference Committee. Crit Care Med 1992; 20: $867-874$.

13. Members of the American College of Chest Physicians/Society of Critical Care Medicine Consensus Conference Committee. Crit Care Med 1992; 101: $1644-1655$.

14. Nakae H, Endo S, Yamada Y, Inada K. Bound and soluble adhesion molecule and cytokine levels in patients with severe burns. Burns 2000; 26: $139-144$.
15. Demling R, LaLonde C, Saldinger P, Knox J. Multiple organ dysfunction in the surgical patient: Pathophysiology, prevention, and treatment. Curr Problems Surg 1993; 4: 348-414.

16. Nakae H, Endo S, Inada K, Yoshida M. Chronological changes in the complement system in sepsis. Surg Today 1996; 26: 225-229.

17. Yao YM, Redl H, Bahrami S, Schlag G. The inflammatory basis of trauma/shock-associated multiple organ failure. Inflamm Res 1998; 47: 201-210.

18. Nakae H, Endo S, Inada K, Takakuwas T, Kasai T. Changes in adhesion molecule levels in sepsis. Res Commun Mol Pathol Pharmacol 1996; 3: 329-338.

19. Demling RH, LaLonde C. Early burn excision attenuates the postburn lung and systemic response to endotoxin. Surgery 1990; 108: 28-35.

20. Deveci M, Eski M, Sengezer M, Kisa U. Effects of cerium nitrate bathing and prompt burn wound excision on IL-6 and TNF-alpha levels in burned rats. Burns 2000; 26: 41-45.

21. Chai J, Sheng Z, Yang H, Diao L, Li L. Successful treatment of invasive burn wound infection with sepsis in patients with major burns. Chin Med J 2000; 113: 1142-1146.

22. Papini RP, Wilson AP, Steer JA, Hill G, McGrouther DA, Parkhouse N. Plasma concentrations of tumour necrosis factor-alpha and interleukin-6 during burn wound surgery or dressing. Br J Plast Surg 1997; 50: $354-$ 361.

23. White J, Thomas J, Maass DL, Horton JW. Cardiac effects of burn injury complicated by aspiration pneumonia-induced sepsis. Am J Physiol Heart Circ Physiol 2003; 285: 47-58.

24. Ljunghusen $\mathrm{O}$, Berg S, Hed J, et al. Transient endotoxemia during burn wound revision causes leukocyte beta 2 integrin up-regulation and cytokine release. Inflammation 1995; 19: 457-468.

25. Schinkel C, Faist E, Zimmer S, et al. Kinetics of circulating adhesion molecules and chemokines after mechanical trauma and burns. Eur J Surg 1996; 162: 763-768.

\section{Received 12 April 2004}

Accepted 14 May 2004 


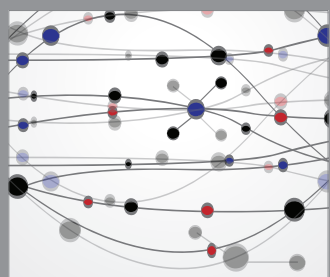

The Scientific World Journal
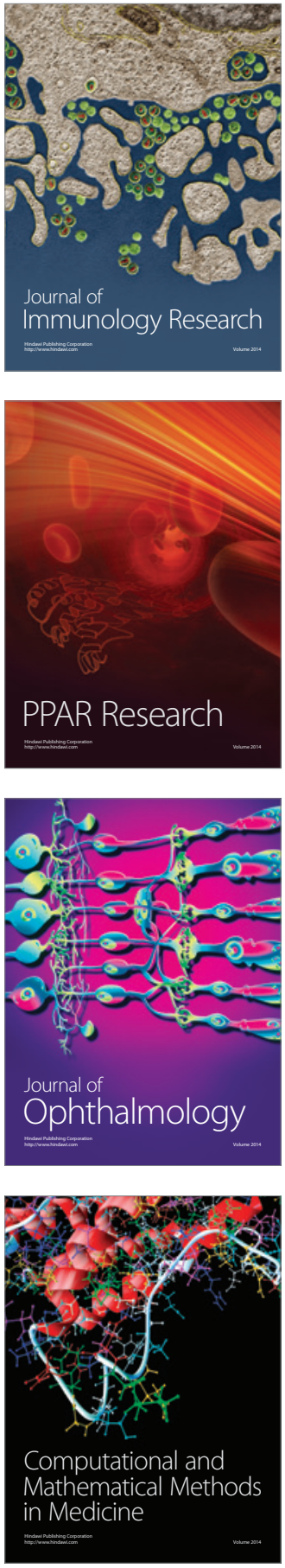

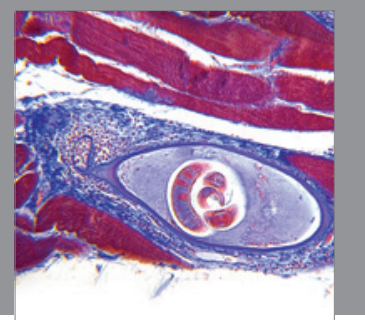

Gastroenterology

Research and Practice
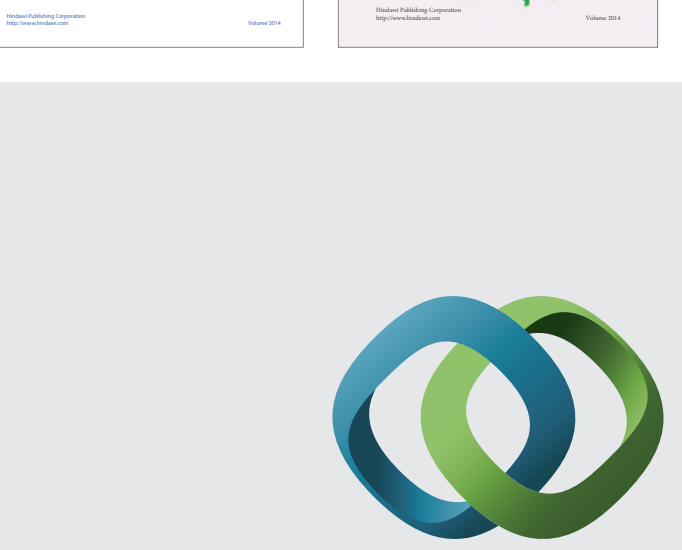

\section{Hindawi}

Submit your manuscripts at

http://www.hindawi.com
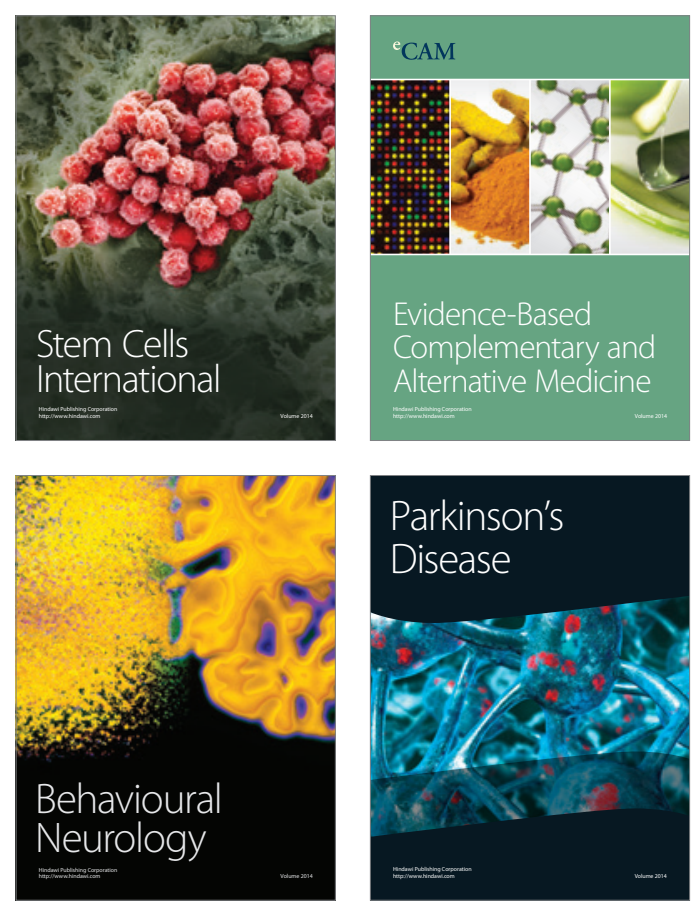

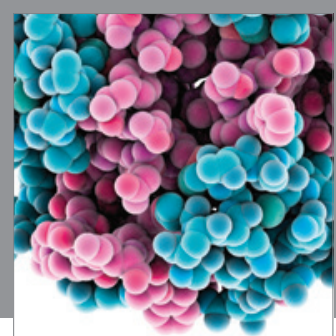

Journal of
Diabetes Research

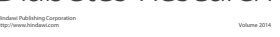

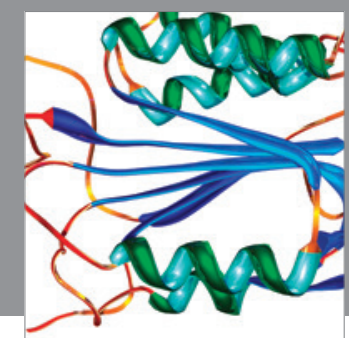

Disease Markers
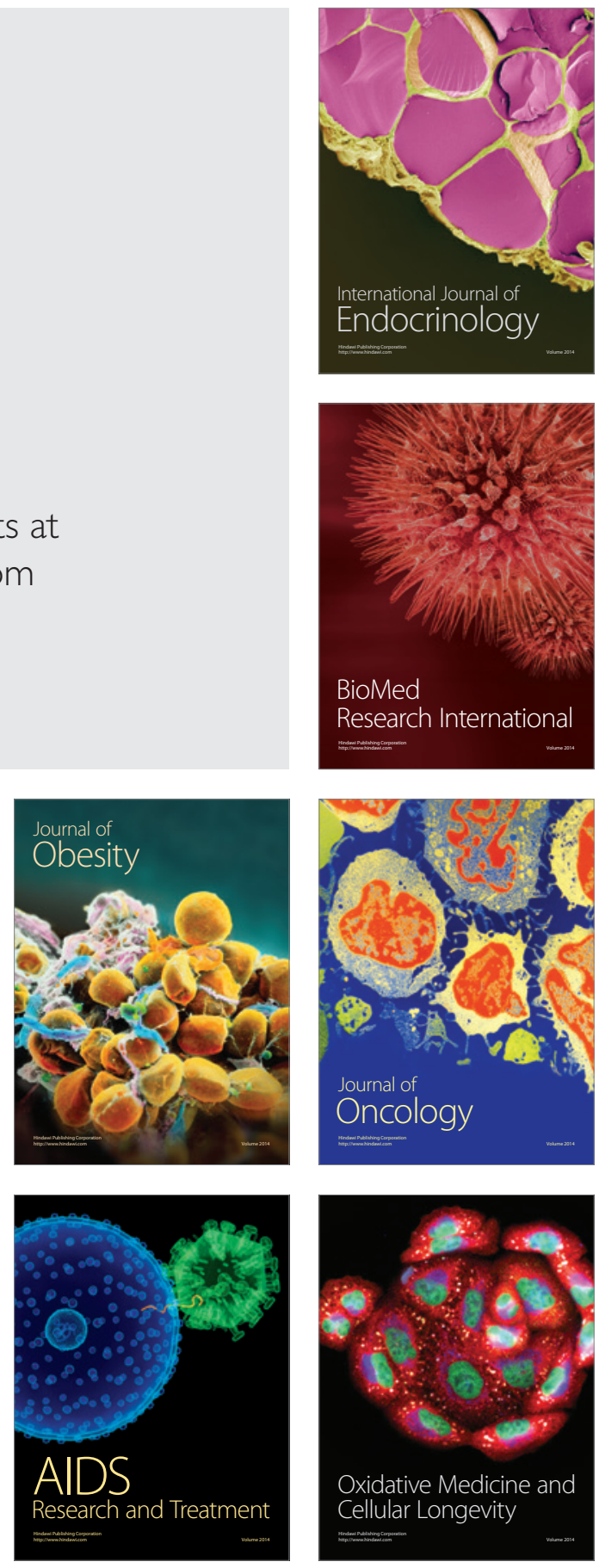